\title{
A Unified Strategy to Access Trans-Syn-Fused Drimane Meroterpe- noids: Chemoenzymatic Total Syntheses of Polysin, $N$-Acetyl-Polyve- oline and the Chrodrimanins
}

\author{
Fuzhuo Li, Hans Renata*,§ \\ §Department of Chemistry, The Scripps Research Institute, 130 Scripps Way, Jupiter, FL 33458, USA
}

ABSTRACT: Trans-syn-fused drimane meroterpenoids are unique natural products that arise from contra-thermodynamic polycyclizations of their polyene precursors. Herein we report the first total syntheses of four trans-syn-fused drimane meroterpenoids, namely polysin, $\mathrm{N}$-acetyl-polyveoline, chrodrimanin $\mathrm{C}$ and verruculide A in 7-18 steps from sclareolide. The trans-syn-fused drimane unit is accessed through an efficient acid-mediated C9 epimerization of sclareolide. Subsequent applications of enzymatic $\mathrm{C}-\mathrm{H}$ oxidation and contemporary annulation methodologies install the requisite C3 hydroxyl group and enable rapid generation of structural complexity to provide concise access to these natural products.

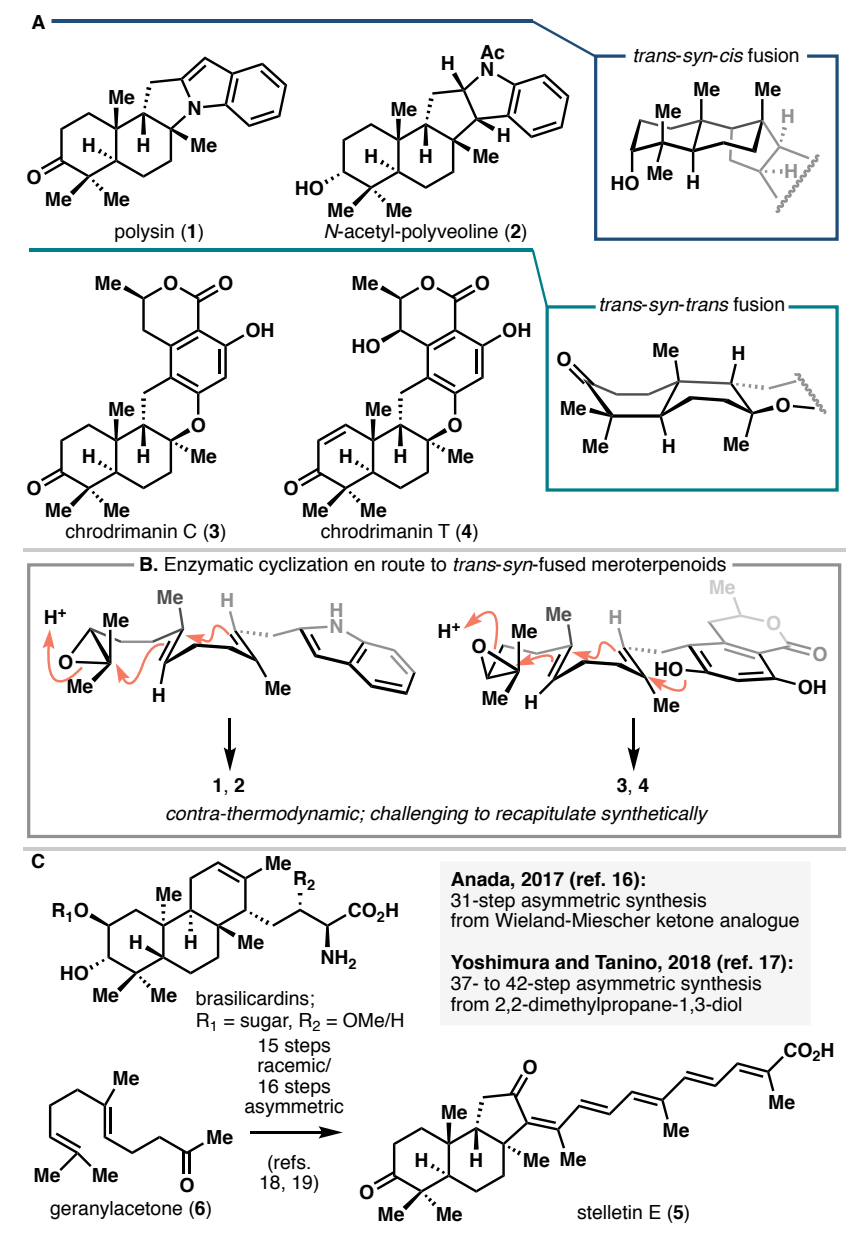

Figure 1. A. Select examples of trans-syn-fused drimane meroterpenoids. B. Biosynthesis of trans-syn-fused meroterpenoids. C. Prior total syntheses of trans-syn-fused terpenoids.

Meroterpenoids are a highly diverse class of natural products that arise in nature from hybrid terpenoid/non-terpenoid biosynthetic pathways. ${ }^{1}$ One highly prevalent motif in many meroterpenoids is the C3-oxidized drimane substructure, which forms the central core of many families including the 3,5-dimethylorsellinic acid-derived fungal meroterpenoids ${ }^{2}$ and the sesquiterpenyl indoles. ${ }^{3}$ Biosynthetically, this motif is produced through the cyclization of a linear polyisoprene-derived epoxide precursor by various terpene cyclases. Compelling literature evidence ${ }^{3,4,5}$ has suggested that these enzymes are capable of pre-organizing their respective substrates in specific conformations to generate products with unique ring topologies and stereoconfigurations, which ultimately contribute to the immense structural diversity of the meroterpenoids.

Among the possible polycyclization product topologies, the alltrans configuration is the most favored thermodynamically as it allows the fused cyclohexane rings to adopt an all-chair conformation. However, there exists a subset of drimane-containing meroterpenoids that possess alternative ring fusions (Figure $1 \mathrm{~A})$, such as the trans-syn-cis-fusion found in polysin ${ }^{6}(\mathbf{1})$ and $\mathrm{N}$-acetyl-polyveoline ${ }^{7}(2)$ and the trans-syn-trans-fusion found in the chrodrimanins ${ }^{8,9}$ (e.g., 3 and 4). Access to thermodynamically disfavored ring fusions in these meroterpenoids is made possible by the ability of the respective cyclases to generate the less stable, boat-like transition state during their reactions (Figure 1B). Such conformational requirements have proven to be prohibitive for synthetic recapitulation as attempts to effect biomimetic cyclizations to prepare trans-synfused drimanes have been met with limited to no success. ${ }^{10,11}$ A synthetic approach towards polyveoline featuring an indoleterminated polyene cyclization failed to overcome the innate thermodynamic preference of the substrate and resulted in exclusive formation of the undesired all-trans product. ${ }^{10}$ Though the use of substrates with alternative olefin placement or geometry has garnered some success, ${ }^{12,13}$ these approaches have resulted in either sub-optimal diastereoselectivity or low yields for the desired products. 
A

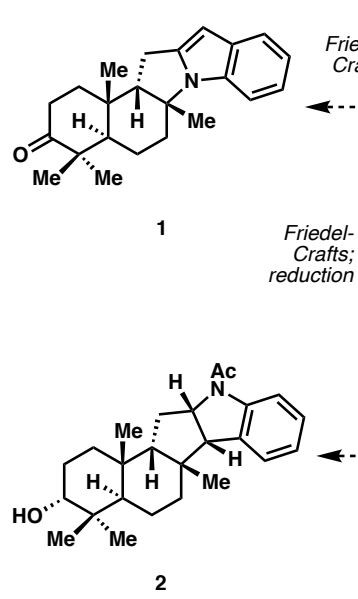

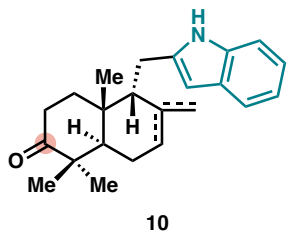

10
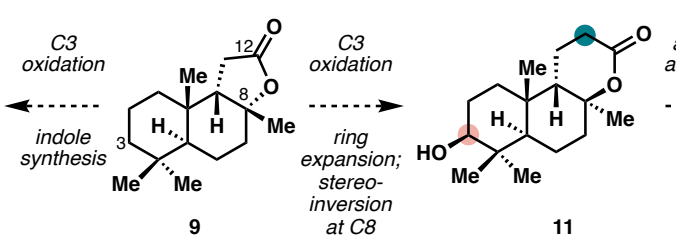

aromatic annulation precedent for C9 epimerization of sclareolide (ref. 20)
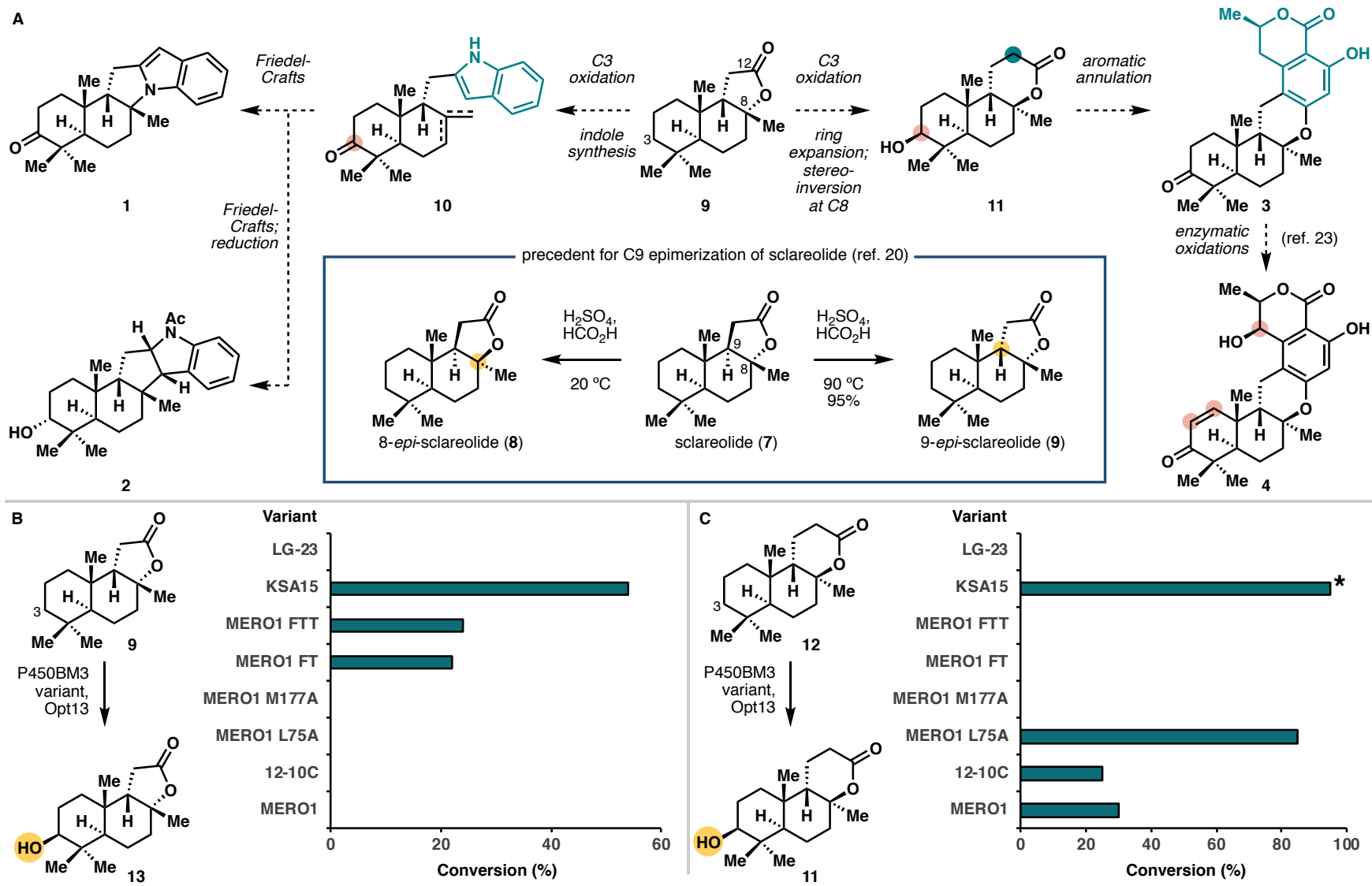

Figure 2. A. Synthetic strategy to access polysin, $N$-acetyl-polyveoline and the chrodrimanins from $\mathbf{9}$, which could be obtained via C9 epimerization of sclareolide (7). B. Screening of $\mathrm{P}_{5} 50_{\text {вмз }}$ variants in our collection for the $\mathrm{C} 3$ hydroxylation of $\mathbf{9}$. C. Screening of $\mathrm{P}^{4} \mathrm{~B}_{\mathrm{Bм}}$ variants in our collection for the $\mathrm{C} 3$ hydroxylation of 12. See Supporting Information for the identities of the variants tested. Reaction conditions for enzymatic hydroxylation were: 9 or $12(5.0 \mathrm{mM}), \mathrm{NADP}^{+}(1.0 \mathrm{mM}), \mathrm{NaHPO}_{3}(100 \mathrm{mM})$, clarified lysate of $E$.

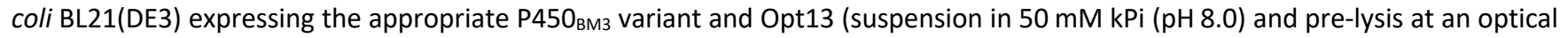
density of 30 , measured at a wavelength of $600 \mathrm{~nm}$ ) for $20 \mathrm{~h}$ at $20^{\circ} \mathrm{C}$. *additional regioisomers were detected in the product mixture.

In parallel with the above efforts, several groups have sought to harness the power of terpene cyclases to biocatalytically access trans-syn-fused terpenoids. Since van Tamelen's landmark study on a cyclase from rat liver, ${ }^{14}$ several reports have demonstrated the feasibility of this approach. Virgil and coworkers were able to use an unnatural oxidosqualene derivative in an enzymatic polycyclization to access the isomalabaricane tricyclic core ${ }^{15}$ and more recently, a collaborative work by the Porco and Abe laboratories ${ }^{5}$ showcased the utility of several fungal cyclases in constructing unnatural meroterpenoids with unusual ring fusions from synthetic substrates. These demonstrations notwithstanding, the approach suffers from low material throughput arising from the inefficiency of the enzymatic reaction and the difficulty in obtaining large quantities of the membrane-bound enzymes.

In the context of target-oriented chemical synthesis, only three total syntheses of trans-syn-fused drimane terpenoids have been reported thus far (Figure 1C). Two of these syntheses ${ }^{16,17}$ pertain the brasilicardin natural products and involved lengthy synthetic sequences to generate the key trans-syn-trans-fused tricyclic intermediates. More recently, a landmark synthesis of the isomalabaricanes (e.g., stellettin E, 5) by the Sarlah group has enabled initial structure-activity relationship studies on the cytotoxicity of the scaffold. ${ }^{18,19}$ To complement the aforementioned approaches, we sought to develop an alternative strategy to collectively prepare trans-syn-fused drimane meroterpenoids through the use of a chiral pool approach. This report discloses the development of a unified strategy to access both trans-syn-cis- and trans-syn-trans-fused drimane meroterpenoids from sclareolide that culminates in the first total syntheses of polysin, $\mathrm{N}$-acetyl-polyveoline, chrodrimanin $\mathrm{C}$ and verruculide $A$. To the best of our knowledge, this is the first reported de novo constructions of trans-syn-cis-fused perhydrobenz [e]indene and trans-syn-trans-fused dodecahydro- $1 \mathrm{H}$ benzo[f]chromene frameworks. This work was made possible by the use of an underexplored epimerization reaction on sclareolide, ${ }^{20}$ which was combined with enzymatic $\mathrm{C}-\mathrm{H}$ oxidations and efficient ring annulations to complete the divergent syntheses. We anticipate that the strategy delineated herein will find a broad range of applications in the preparation of other drimane terpenoids with unusual ring fusions.

As noted above, our synthetic strategy was predicated upon the ability of sclareolide (7) to undergo facile epimerization at its $\mathrm{C} 8$ and $\mathrm{C} 9$ positions. Prior report from Ohloff ${ }^{20}$ (Figure 2A, inset) showed that treatment of $\mathbf{7}$ with mineral acid at room temperature could readily afford the C8-epimerized product 
(8). Alternatively, the C9-epi product (9) was observed as the major product at elevated temperature, likely via elimination to the corresponding $\mathrm{C} 8-\mathrm{C} 9$ olefin, followed by re-protonation from the $\beta$-face at $\mathrm{C} 8$ and quenching of the $\mathrm{C} 9$ carbocation from the $\alpha$-face by the pendant carboxylic acid. In our hands, this transformation could be routinely conducted on multigram scale with $95 \%$ yield. With the C9 stereocenter established, access to polysin and $\mathrm{N}$-acetyl-polyveoline could be accomplished through selective $\mathrm{C}-\mathrm{H}$ oxidation at $\mathrm{C} 3$ and the appendage of a pendant indole unit (Figure 2A). We envisioned introducing the former through enzymatic $\mathrm{C}-\mathrm{H}$ hydroxylation $^{21,22}$ and the latter through ring synthesis by leveraging the C12 carbonyl as a chemical handle. Adaptation of this idea to access the chrodrimanin series would necessitate the invention of a synthetic sequence to construct the C-ring pyran while also inverting the stereochemistry at $\mathrm{C} 8$. While the general pyran structure could be prepared via a one-carbon homologation, the stereoinversion at $\mathrm{C} 8$ was expected to be non-trivial as it would result in an $\mathrm{A} / \mathrm{B} / \mathrm{C}$-ring connectivity that forces the $B$-ring to adopt the energetically-unfavored twist-boat conformation. Nevertheless, if this transformation could be realized, an efficient synthesis of chrodrimanin $\mathrm{C}$ would ensue through subsequent use of the $\mathrm{C}$-ring lactone as a chemical handle in an aromatic annulation sequence. Finally, enzymatic conversion of $\mathbf{3}$ to $\mathbf{4}$ through a series of in vitro reactions has previously been reported by Matsuda, Abe and co-workers. ${ }^{23}$

Scheme 1. Chemoenzymatic total synthesis of polysin (1) and $\mathrm{N}$-acetyl-polyveoline (2) via enzymatic C-H oxidation of $\mathbf{9 .}$
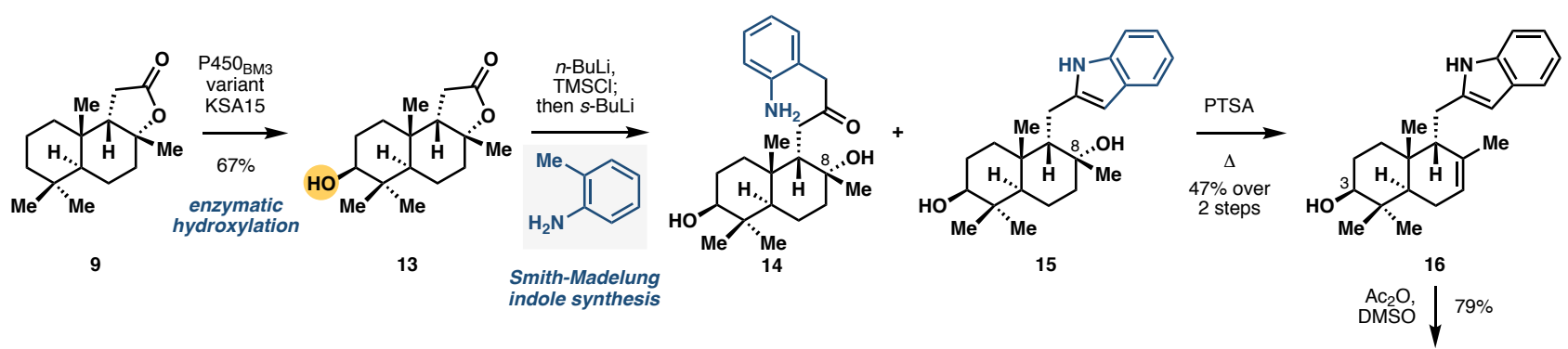
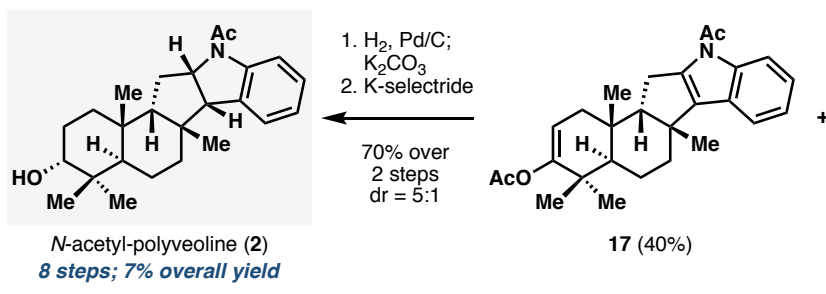

In light of our previous work in the synthesis of $\alpha$-pyrone meroterpenoids from 7, a route involving enzymatic C3 oxidation of 7 with variants of $\mathrm{P}^{4} 5 \mathrm{O}_{\mathrm{BM}},{ }^{22}$ followed by epimerization at $\mathrm{C} 9$ was initially considered. However, preliminary forays into this route showed that the $\mathrm{C} 3$ alcohol is incompatible with strong acids, even in its protected form. As a workaround, we decided to investigate the feasibility of performing enzymatic $\mathrm{C}-\mathrm{H}$ oxidation on lactones 9 and 12, which was prepared in seven steps from 9 (vide infra). Despite the high structural similarities of 9 and $\mathbf{1 2}$ to 7, it is widely accepted that even minor alterations in substrate structure could result in dramatic changes in reactivity in enzymatic transformations. Gratifyingly, initial screening of a subset of our $\mathrm{P}^{4} 5 \mathrm{O}_{\mathrm{BM}}$ library revealed a few variants with C3 hydroxylation activity on 9 (Figure 2B). Variant $\mathrm{KSA} 15,{ }^{24}$ previously developed by Reetz and co-workers for steroid hydroxylation, showed the highest conversion (54\%) among all the library members tested. Following an analogous screening with lactone $\mathbf{1 2}$, variant MERO1 L75A, previously developed in our laboratory for the synthesis of oxidized meroditerpenoids, ${ }^{22}$ was identified to be the optimal enzyme to hydroxylate 12 at C3 (Figure 2C). While variant KSA15 provided higher conversion (95\%) in its reaction with $\mathbf{1 2}$, additional product regioisomers could be detected. Thus, we elected to perform subsequent $\mathrm{C}-\mathrm{H}$ oxidation scale-up with MERO1 L75A.
With the above results in hand, we set our sights toward establishing a concise access to polysin and $\mathrm{N}$-acetyl-polyveoline (Scheme 1). Preparative scale enzymatic hydroxylation of $\mathbf{9}$ provided alcohol 13 with $67 \%$ yield, which was subjected to a Smith-modified Madelung indole synthesis ${ }^{25}$ to provide a mixture of two adducts, 14 and 15. Treatment of this mixture with $p$-toluenesulfonic acid (PTSA) effected complete formation of the indole nucleus with concomitant dehydration of the tertiary alcohol at C8. In light of its potential incompatibility with acidic conditions needed for the subsequent cyclization step, oxidation of the C3 alcohol at this stage was deemed strategic and was accomplished using Albright-Goldman protocols. ${ }^{26}$ Following an extensive screening of Lewis acids (see Supporting Information Table S4), we arrived at the use of MK-10 under microwave heating to generate a mixture of Friedel-Crafts adducts. As the $C$-cyclized product was observed to be unstable, an in-situ capping approach with $\mathrm{Ac}_{2} \mathrm{O}$ was devised to deliver a mixture of enol ethers 17 and 18 in $40 \%$ and $25 \%$ yields respectively under telescoped procedure. Routine saponification of $\mathbf{1 8}$ completed the synthesis of polysin (1) in seven steps from 7. Conversely, $\mathbf{1 7}$ was subjected to hydrogenation in the presence of palladium on carbon, followed by a diastereoselective reduction with $\mathrm{K}$-selectride to complete the synthesis of $\mathrm{N}$-acetyl-polyveoline (2) in eight steps from sclareolide (7). While indole hydrogenation typically requires high $\mathrm{H}_{2}$ 
pressure, 17 could be reduced with just 1 atm of $\mathrm{H}_{2}$ pressure and we surmised that a strain release phenomenon might be in play to facilitate such hydrogenation under mild conditions.

Synthesis of $\mathbf{1 2}$ from $\mathbf{9}$ was initiated by thermal opening of the latter's lactone ring to generate acid $\mathbf{1 9}$ (Scheme 2). A threestep Arndt-Eistert homologation of $\mathbf{1 9}$ afforded methyl ester 20, which was subjected to Mukaiyama hydration. Prior studies by Shenvi and co-workers ${ }^{27}$ in the synthesis of bilobalide showed that the reaction solvent polarity could have significant effects on the product diastereoselectivity. Based on this precedent, we undertook extensive optimization of this step by varying the reaction solvent, temperature, catalyst and reductant (see Supporting Information Table S5). Unfortunately, no marked increase in diastereoselectivity was observed in all tion and $6 \pi$ electrocyclization.

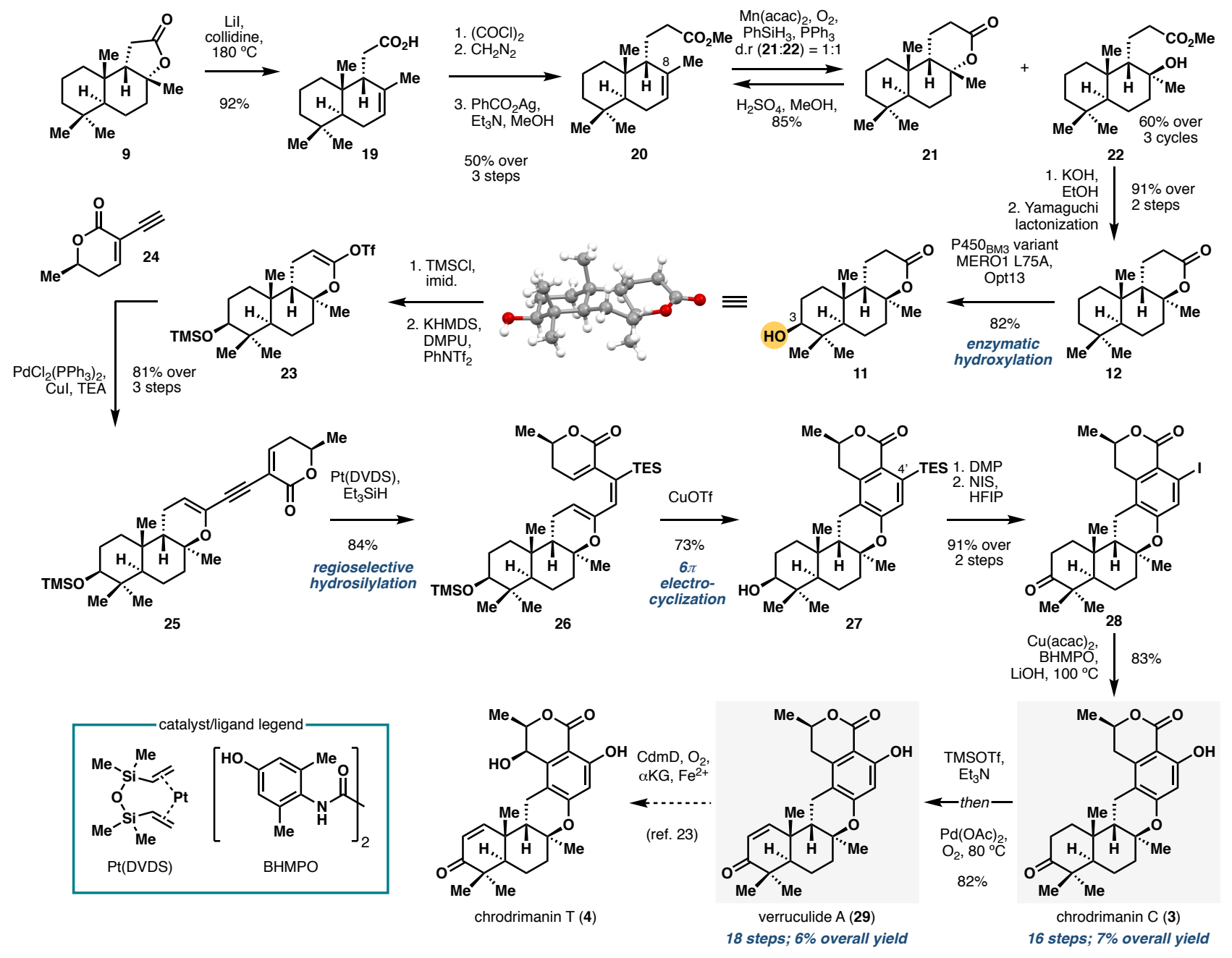

Drawing inspiration from the syntheses of arene-containing terpenoids by $\mathrm{Li}$ and co-workers, ${ }^{28,29}$ we sought to construct the central arene ring of the chrodrimanins through a $6 \pi$ electrocyclization of the corresponding triene precursor. Toward this goal, the $\mathrm{C} 3$ alcohol of $\mathbf{1 1}$ was temporarily protected as the trimethylsilyl (TMS) ether and the C-ring lactone was converted to the corresponding vinyl triflate (compound 23). Sonogashira coupling of $\mathbf{2 3}$ with alkyne $\mathbf{2 4}$, synthesized in 6 steps conditions tried and under the best set of conditions, a diastereomeric ratio of 1:1 at C8 was obtained. At this stage, the desired tertiary alcohol diastereomer $\mathbf{2 2}$ was saponified and converted to trans-syn-trans-fused lactone $\mathbf{1 2}$ through the use of Yamaguchi's reagent. To improve material throughput, the unwanted diastereomer $\mathbf{2 1}$ could be recycled into the sequence by simple methyl ester formation to regenerate $\mathbf{2 0}$ along with its olefin regioisomer. After three cycles, a combined $60 \%$ isolated yield of $\mathbf{2 2}$ could be achieved. Enzymatic hydroxylation of 12 with $\mathrm{P}^{4} 50_{\mathrm{BM}}$ variant MERO1 L75A was next conducted on preparative scale to provide alcohol $\mathbf{1 1}$ in $82 \%$ yield. The structure of this compound was verified by $\mathrm{X}$ ray diffraction analysis, which prominently revealed the twistboat configuration of the B-ring.

Scheme 2. Chemoenzymatic total synthesis of $\mathbf{3}$ and $\mathbf{2 9}$ featuring enzymatic C-H oxidation of 12, regioselective alkyne hydrosilyla-

from $(R)$-methyl 3-hydroxybutanoate (see Supporting Information), delivered dienyne $\mathbf{2 5}$ in $67 \%$ yield over 3 steps from alcohol 11. With the goal of introducing a suitable functional handle for subsequent phenol formation, an alkyne hydrosiIylation approach was pursued. Previous work by Ferreira and co-workers ${ }^{30}$ showed that the regioselectivity of alkyne hydrosilylation is predominantly dictated by electronic effects whereby hydride delivery would take place at the $s p$ carbon 
that is further away from electron-withdrawing group. Indeed, treatment of alkyne $\mathbf{2 5}$ with $\mathrm{Et}_{3} \mathrm{SiH}$ in the presence of catalytic Pt(DVDS) successfully provided the desired hydrosilylation product $\mathbf{2 6}$ as a single regioisomer.

Following precedent by $\mathrm{Li}$, Nicolaou and co-workers, ${ }^{31}$ a $6 \pi$ electrocyclization/aromatization sequence could be effected to generate arene $\mathbf{2 7}$. In agreement with their work, the use of CuOTf as a Lewis acid promoter was found to improve the yield of the reaction ( $73 \%$ isolated yield) while also effecting a concomitant hydrolysis of the TMS ether at C3. While oxidation of the $\mathrm{C} 3$ alcohol to the corresponding ketone proceeded uneventfully, attempts to effect a Fleming-Tamao oxidation ${ }^{32}$ to convert $\mathbf{2 7}$ to the corresponding phenol were met with failure. Similar outcomes were obtained when alternative silanes at C4' were tested in the reaction. Earlier iterations of the route featuring a late-stage $s p^{2} \mathrm{C}-\mathrm{H}$ oxidation at $\mathrm{C}^{\prime}$ using Ru cataly$\mathrm{sis}^{33}$ or peroxide-based reagents ${ }^{34,35}$ also failed to deliver the desired product. As a workaround, silane $\mathbf{2 7}$ was first subjected to desilylative iodination with NIS to provide 28. Following screening of several reported conditions for haloarene hydroxylation, access to chrodrimanin C (3) could be realized through the use of $\mathrm{Cu}(\mathrm{acac})_{2}$ and $N, N^{\prime}$-bis(4-hydroxyl-2,6-dimethylphenyl)oxalamide (BHMPO) on 28. ${ }^{36}$ This method, initially reported by $\mathrm{Ma}$ and co-workers, proved superior to $\mathrm{Pd}$-based hydroxylation methods ${ }^{37,38}$ and with slight modifications to the originally reported conditions, the desired phenol product could be obtained in $83 \%$ isolated yield. A-ring desaturation of 3 proceeded uneventfully under standard Saegusa conditions to deliver verruculide A (29) in $82 \%$ yield. Interestingly, TMS ether formation at the phenolic $\mathrm{OH}$ was not observed in this reaction, likely due to the presence of an intramolecular hydrogen bonding with the neighboring lactone. Overall, this sequence provided a 16-step synthesis of chrodrimanin C (3) and a 18-step synthesis of verruculide A (29) from sclareolide (7), respectively. As noted earlier, the biosynthetic pathway towards the chrodrimanins was recently elucidated by Matsuda, Abe and co-workers ${ }^{23}$ and we anticipate that future work involving incorporation of some of the enzymes from the pathway would allow for a rapid chemoenzymatic diversification of the scaffold to provide a wider range of synthetic chrodrimanins.

This work reports the development of a chiral-pool-based strategy for the asymmetric synthesis of trans-syn-fused drimane meroterpenoids. Two enabling features in the synthesis are the strategic use of an acid-mediated C9-epimerization of sclareolide to generate the general trans-syn-fused architecture of these natural products and the ability to perform regioselective $\mathrm{C}-\mathrm{H}$ oxidations on different key synthetic intermediates at their $\mathrm{C} 3$ position by relying on a small pool of $\mathrm{P}^{450_{\mathrm{BM}}}$ biocatalysts. By combining these features with contemporary annulation methodologies, the first total syntheses of polysin, $\mathrm{N}$-acetyl-polyveoline, chrodrimanin $\mathrm{C}$ and verruculide A could be realized. The route disclosed herein lays the foundation for future synthetic access to other unusually-cyclized meroterpenoids and their unnatural derivatives to facilitate a more thorough investigation into their pharmacology.

\section{Supporting Information}

Experimental details, analytical data, ${ }^{1} \mathrm{H}$ and ${ }^{13} \mathrm{C}$ NMR data (PDF)

\section{AUTHOR INFORMATION}

\section{Corresponding Author}

*hrenata@scripps.edu.

\section{Author Contributions}

The manuscript was written through contributions of all authors. / All authors have given approval to the final version of the manuscript.

\section{Funding Sources}

This work is supported, in part, by the National Science Foundation CAREER Award (H.R. grant \#1945468) and the National Institutes of Health Grant S10 OD021550 (for 600 MHz NMR instrumentation).

\section{Notes}

The authors declare no competing financial interests.

\section{ACKNOWLEDGMENT}

Plasmid encoding for $\mathrm{P} 450_{\mathrm{BM} 3}$ variant $12-10 \mathrm{C}$ was generously provided by F. H. Arnold. We acknowledge funding from the National Science Foundation CAREER Award (H.R. grant \#1945468) and support from the National Institutes of Health for $600 \mathrm{MHz}$ NMR instrumentation (grant S10 OD021550). We thank Dr. Xiao Zhang for generating the $\mathrm{P} 450_{\mathrm{BM} 3}$ variants examined in this work. We are grateful to the Shen and Bannister labs for generous access to their reagents and instrumentation.

\section{REFERENCES}

1. Gozari, M.; Alborz, M.; El-Seedi, H. R.; Jassbi, A. R. Chemistry, biosynthesis and biological activity of terpenoids and meroterpenoids in bacteria and fungi isolated from different marine habitat. Eur. J. Med. Chem. 2021, 210, 112957.

2. Matsuda, Y.; Abe, I. Biosynthesis of fungal meroterpenoids. Nat. Prod. Rep. 2016, 33, 26-53.

3. Marcos, I. S.; Moro, R. F.; Costales, I.; Basabe, P.; Díez, D. Sesquiterpenyl indoles. Nat. Prod. Rep. 2013, 30, 15091526.

4. Matsuda, Y.; Wakimoto, T.; Mori, T.; Awakawa, T.; Abe, I. Complete biosynthetic pathway of anditomin: Nature's sophisticated synthetic route to a complex fungal meroterpenoid. J. Am. Chem. Soc. 2014, 136, 15326-15336.

5. Mitsuhashi, T.; Barra, L.; Powers, Z.; Kojasoy, V.; Cheng, A.; Yang, F.; Taniguchi, Y.; Kikuchi, T.; Fujita, M.; Tantillo, D. J.; Porco, J. A., Jr; Abe, I. Exploiting the potential of meroterpenoid cyclases to expand the chemical space of fungal meroterpenoids. Angew. Chem. Int. Ed. 2020, 59, 23772-23781.

6. Ngantchou, I.; Nyasse, B.; Denier, C.; Blonsk, C.; Hannert, V.; Schneider, B. Antitrypanosomal alkaloids from Polyalthia suaveolens (Annonaceae): Their effects on three selected glycolytic enzymes of Trypanosoma brucei. Bioorg. Med. Chem. Lett. 2010, 20, 3495-3498.

7. Kouam, S. F.; Ngouonpe, A. W.; Lamshöft, M.; Talontsi, F. M.; Bauer, J. O.; Strohmann, C.; Ngadjui, B. T.; Laatsch, H.; Spiteller, M. Indolosesquiterpene alkaloids from the Cameroonian medicinal plant Polyalthia oliveri (Annonaceae). Phytochemistry 2014, 105, 52-59.

\section{ASSOCIATED CONTENT}


8. Hayashi, H.; Oka, Y.; Kai, K.; Akiyama, K. A new meroterpenoid, chrodrimanin C, from YO-2 of Talaromyces sp. Biosci. Biotechnol. Biochem. 2012, 76, 745-748.

9. Yamazaki, H.; Nakayama, W.; Takahashi, O.; Kirikoshi, R.; Izumikawa, Y.; Iwasaki, K.; Toraiwa, K.; Ukai, K.; Rotinsulu, H.; Wewengkang, D. S.; Sumilat, D. A.; Mangindaan, R. E. P.; Namikoshi, M. Verruculides $A$ and $B$, two new protein tyrosine phosphatase $1 \mathrm{~B}$ inhibitors from an Indonesian ascidian-derived Penicillium verruculosum. Bioorg. Med. Chem. Lett. 2015, 25, 3087-3090.

10. Mirand, C.; de Maindreville, M. D.; Cartier, D.; Lévy, J. Nbenzene-sulfonyl-indole as terminator in a biomimetic polyene cyclization: synthesis of a pentacyclic indolosesquiterpene. Tetrahedron Lett. 1987, 28, 3565-3568.

11. Raeppel, F.; Weibel, J.-M.; Heissler, D. Synthesis of the trans-syn-trans perhydrobenz[e]indene moiety of the stellettins and of the stelliferins. Tetrahedron Lett. 1999, 40, 6377-6381.

12. Yee, N. K. N.; Coates, R. M. Total synthesis of (+)-9,10-synand (+)-9,10-anti-copalol via epoxy trienylsilane cyclizations. J. Org. Chem. 1992, 57, 4598-4608.

13. Powers, Z.; Scharf, A.; Cheng, A.; Yang, F.; Himmelbauer, M.; Mitsuhashi, T.; Barra, L.; Taniguchi, Y.; Kikuchi, T.; Fujita, M.; Abe, I.; Porco, J. A., Jr. Biomimetic synthesis of meroterpenoids by dearomatization-driven polycyclization. Angew. Chem. Int. Ed. 2019, 58, 16141-16146.

14. Van Tamelen, E. E.; Sharpless, K. B.; Hanzlik, R.; Clayton, R. B.; Burlingame, A. L.; Wszolek, P. C. Enzymic cyclization of trans, trans, trans-18, 19-dehydrosqualene 2, 3-oxide. J. Am. Chem. Soc. 1967, 89, 7150-7151.

15. Rosner, K. E. Approaches to the synthesis of stelliferin, a marine isomalabaricane triterpene. Ph.D. Dissertation, Massachusetts Institute of Technology, Cambridge, MA, 1996.

16. Anada, M.; Hanari, T.; Kakita, K.; Kurosaki, Y.; Katsuse, K.; Sunadoi, Y.; Jinushi, Y.; Takeda, K.; Matsunaga, S.; Hashimoto, S. Total synthesis of brasilicardins A and C. Org. Lett. 2017, 19, 5581-5584.

17. Yoshimura, F.; Itoh, R.; Torizuka, M.; Mori, G.; Tanino, K. Asymmetric total synthesis of brasilicardins. Angew. Chem. Int. Ed. 2018, 57, 17161-17167.

18. Boyko, Y.; Huck, C. J.; Sarlah, D. The total synthesis of isomalabaricane triterpenoids. J. Am. Chem. Soc. 2019, 141, 14131-14135.

19. Boyko, Y.; Huck, C. J.; Ning, S.; Shved, A. S.; Yang, C.; Chu, T.; Tonogai, E. J.; Hergenrother, P. J.; Sarlah, D. Synthetic studies on selective, proapoptotic isomalabaricane triterpenoids aided by computational techniques. J. Am. Chem. Soc. 2021, 143, 2138-2155

20. Ohloff, G.; Giersch, W.; Pickenhagen, W.; Furrer, A.; Frei, B. Significance of the geminal dimethyl group in the odor principle of Ambrox ${ }^{\circledR}$. Helv. Chim. Acta 1985, 68, 2022-2029.

21. Zhang, K.; El Damaty, S.; Fasan, R. P450 fingerprinting method for rapid discovery of terpene hydroxylating P450 catalysts with diversified regioselectivity. J. Am. Chem. Soc. 2011, 133, 3242-3245.

22. Li, J.; Li, F.; King-Smith, E.; Renata, H. Merging chemoenzymatic and radical-based retrosynthetic logic for rapid and modular synthesis of oxidized meroterpenoids. Nat. Chem. 2020, 12, 173-179.
23. Bai, T.; Quan, Z.; Zhai, R.; Awakawa, T.; Matsuda, Y.; Abe, I. Elucidation and heterologous reconstitution of chrodrimanin B biosynthesis. Org. Lett. 2018, 20, 7504-7508.

24. Kille, S.; Zilly, F. E.; Acevedo, J. P.; Reetz, M. T. Regio- and stereoselectivity of P450-catalysed hydroxylation of steroids controlled by laboratory evolution. Nat. Chem. 2011, 3, 738-743.

25. Smith, A. B., III; Visnick, M.; Haseltine, J. N.; Sprengeler, P. A. Organometallic reagents in synthesis: A new protocol for construction of the indole nucleus. Tetrahedron 1986, 42, 2957-2969.

26. Zhang, Z.; Xie, S.; Cheng, B.; Zhai, H.; Li, Y. Enantioselective Total Synthesis of (+)-Arboridinine, J. Am. Chem. Soc. 2019, 141, 7147-7154.

27. Demoret, R. M.; Baker, M. A.; Ohtawa, M.; Chen, S.; Lam, C.-C.; Khom, S.; Roberto, M.; Forli, S.; Houk, K. N.; Shenvi, R. A. Synthetic, mechanistic and biological interrogation of Ginkgo biloba chemical space en route to (-)-bilobalide. J. Am. Chem. Soc. 2020, 142, 18599-18618.

28. Yang, P.; Yao, M.; Li, J.; Li, Y.; Li, A. Total synthesis of rubriflordilactone B. Angew. Chem. Int. Ed. 2016, 55, 6964 6968.

29. Yang, P.; Li, J.; Sun, L.; Yao, M.; Zhang, X.; Xiao, W.-L.; Wang, J.; Tian, P.; Sun, H.-D.; Puno, P.-T.; Li, A. Elucidation of the structure of pseudorubriflordilactone $B$ by chemical synthesis. J. Am. Chem. Soc. 2020, 142, 13701-13708.

30. Rooke, D. A.; Ferreira E. M. Platinum-catalyzed hydrosilylations of internal alkynes: harnessing substituent effects to achieve high regioselectivity. Angew. Chem. Int. Ed. 2012, $51,3225-3230$.

31. Bian, M.; Wang, Z.; Xiong, X.; Sun, Y.; Matera, C.; Nicolaou, K.; Li, A. Total syntheses of anominine and tubingensin A, J. Am. Chem. Soc. 2012, 134, 8078-8081.

32. Bracegirdle, S.; Anderson, E. A. Arylsilane oxidation-new routes to hydroxylated aromatics. Chem. Commun. 2010, 46, 3454-3456.

33. Kim, K.; Choe, H.; Jeong, Y.; Lee, J. H.; Hong, S. Ru(II)catalyzed site-selective hydroxylation of flavone and chromone derivatives: the importance of the 5-hydroxyl motif for the inhibition of aurora kinases. Org. Lett. 2015, 17, 2550-2553.

34. Yuan, C.; Liang, Y.; Hernandez, T.; Berriochoa, A.; Houk, K. N.; Siegel, D. Metal-free oxidation of aromatic carbon-hydrogen bonds through a reverse-rebound mechanism. $\mathrm{Na}$ ture 2013, 499, 192-196.

35. Dragan, A.; Kubczyk, T. M.; Rowley, J. H.; Sproules, S.; Tomkinson, N. C. O. Arene oxidation with malonoyl peroxides. Org. Lett. 2015, 17, 2618-2621.

36. Xia, S.; Gan, L.; Wang, K.; Li, Z.; Ma, D. Copper-catalyzed hydroxylation of (hetero)aryl halides under mild conditions. J. Am. Chem. Soc. 2016, 138, 13493-13496.

37. Fier, P.S.; Maloney. K. M. Synthesis of complex phenols enabled by a rationally designed hydroxide surrogate. Angew. Chem. Int. Ed. 2017, 56, 4478-4482.

38. Anderson, K. W.; Ikawa, T.; Tundel, R. E.; Buchwald, S. L. The selective reaction of aryl halides with $\mathrm{KOH}$ : Synthesis of phenols, aromatic ethers, and benzofurans. J. Am. Chem. Soc. 2006, 128, 10694-10695. 


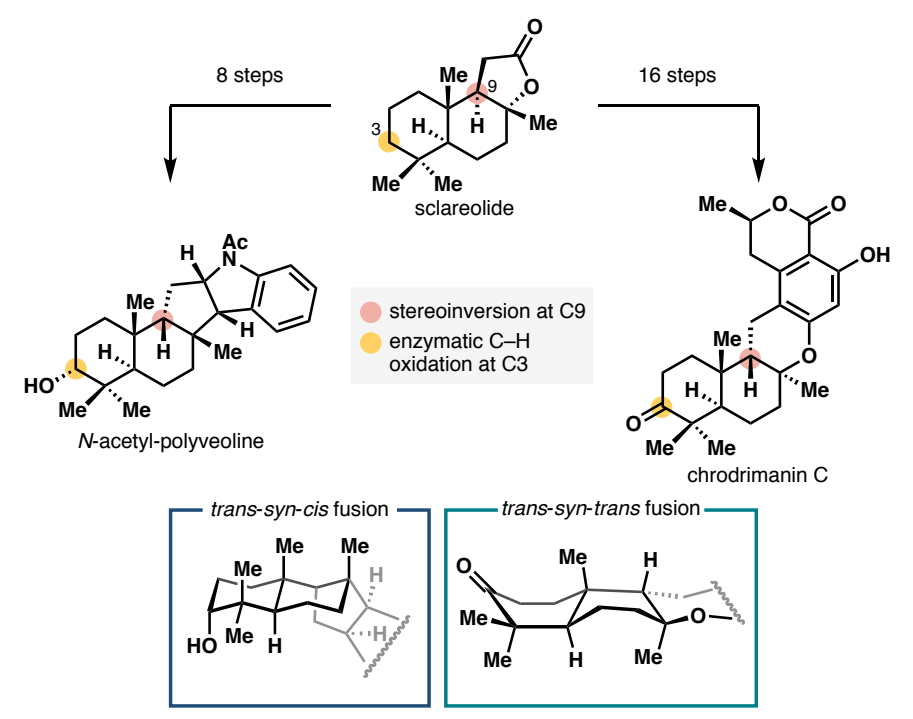

